\title{
Reactive oxygen species and sperm DNA damage in infertile men presenting with low level leukocytospermia
}

Ashok Agarwal ${ }^{1,3^{*}}$, Aditi Mulgund ${ }^{1}$, Saad Alshahrani ${ }^{1,2}$, Mourad Assidi $^{3,4}$, Adel M Abuzenadah ${ }^{3,4}$, Rakesh Sharma ${ }^{1}$ and Edmund Sabanegh ${ }^{1}$

\begin{abstract}
Background: Leukocytes contribute directly and indirectly to reactive oxygen species (ROS) production. Although leukocytospermia is defined as the presence of $\geq 1 \times 10^{6}$ white blood cells $/ \mathrm{mL}(\mathrm{WBC} / \mathrm{mL})$ in a semen sample, the presence of less than $1 \times 10^{6} \mathrm{WBC} / \mathrm{mL}$ (low-level leukocytospermia) can still produce a detectable amount of ROS, impairing sperm function and lowering the chances of pregnancy. Our objective was to assess the effect of low-level leukocytospermia on semen quality, ROS levels, and DNA damage in infertile men.

Methods: Semen samples were examined from 472 patients and divided into 3 groups: no seminal leukocytes; group 2, men with low-level leukoctyospermia (0.1-1.0 $\left.\times 10^{6} \mathrm{WBC} / \mathrm{mL}\right)$; and group 3, frank leukocytospermia, $\left(>1.0 \times 10^{6} . \mathrm{WBC} / \mathrm{mL}\right)$. Semen analysis, leukoctyospermia, reactive oxygen species and DNA fragmentation was tested.

Results: Conventional semen parameters between the 3 groups were similar. Group 2 patients had significantly higher levels of ROS and sperm DNA fragmentation (1839.65 $\pm 2173.57 \mathrm{RLU} / \mathrm{s}$; DNA damage: $26.47 \pm 19.64 \%)$ compared with group 1 (ROS: $1101.09 \pm 5557.54 \mathrm{RLU} / \mathrm{s}$; DNA damage: $19.89 \pm 17.31 \%)$ (ROS: $p=0.002$; DNA damage: $p=0.047$ ). There was no significant difference in ROS levels between groups 2 and 3 .

Conclusions: Patients presenting with low-level leukocytospermia have seminal oxidative stress. Although these patients are not categorized as leukocytospermic by current World Health Organization (WHO) guidelines, these men may benefit by treatment with antibiotics, testing for bacterial cultures, or antioxidant supplements to reduce ROS-induced sperm DNA fragmentation and improve their chances of fertility. The WHO guidelines for leukocytospermia may need to be revised accordingly.
\end{abstract}

Keywords: Male infertility, Low leukocytospermia, Oxidative stress, Reactive oxygen species, DNA fragmentation

\section{Background}

Leukocytospermia refers to the presence of leukocytes in semen. It is defined by the World Health Organization (WHO) as $\geq 1 \times 10^{6} \mathrm{WBC} / \mathrm{mL}$ of semen [1] and is present in $10 \%$ to $20 \%$ of infertile men [2]. The condition can be an indicator of male genital tract infection or inflammation. However, in the absence of infection, it

\footnotetext{
* Correspondence: agarwaa@ccf.org

${ }^{1}$ Center for Reproductive Medicine, Cleveland Clinic, Cleveland, OH 44195, USA

${ }^{3}$ Center of Excellence in Genomic Medicine Research, King AbdulAziz University, Jeddah, Saudi Arabia

Full list of author information is available at the end of the article
}

may originate from etiologies such as cigarette smoking, heavy alcohol use, or age [3-6].

Leukocytes (polymorphonuclear neutrophils and macrophages) have important implications in male fertility in that they produce reactive oxygen species (ROS). At low levels, ROS play a physiologic role [7], but at higher levels, they cause oxidative stress, which overwhelms the physiological mechanisms of sperm and causes damage. This damage has been established to occur via lipid peroxidation of the plasma membrane [8]. After gaining entry into the sperm, ROS target genetic materials, destroying mitochondrial DNA and inhibiting intracellular ATP production $[9,10]$. Without proper ATP production, 
both functionality and sperm motility is affected [11]. As a result, male infertility can occur. Oxidative stress can also decrease success rates of assisted reproduction procedures such as in vitro fertilization (IVF) and intracytoplasmic sperm injection (ICSI) [12,13].

The WHO threshold for leukocytospermia has previously been challenged as being too high due to the detrimental effects seminal leukocytes have on sperm at lower levels [14-17]. Leukocyte counts lower than $1.0 \times 10^{6}$ $\mathrm{WBC} / \mathrm{mL}$ (low-level leukocytospermia) has been shown to cause a significant decrease of motility and DNA integrity [15]. In addition, previous studies have reported high bacterial counts at low levels of leukocytospermia [14], abnormal sperm morphology at levels of leukocytospermia as low as $0.5 \times 10^{6} \mathrm{WBC} / \mathrm{mL}$ [18], and impaired sperm motility with significantly increased levels of cytokines IL-6 and IL-8 in semen with moderately increased leukocytes [19].

Therefore, our objective was to assess the effects of low-level leukocytospermia on semen quality and oxidative stress markers (ROS levels and DNA damage) in infertile men in an effort to examine whether the WHO definition of leukocytospermia should be lowered.

\section{Methods}

\section{Subjects}

This study was approved by Cleveland Clinic review board, and all patients provided informed consent. This observational study used semen samples collected in accordance with a prospective protocol. Between January 2012 and April 2013, 472 infertile patients presenting to the Cleveland Clinic Center for Male Fertility underwent assessment, which included semen analysis. Patients were excluded if they had azoospermia. The following clinical data were recorded: age, body mass index (BMI), volume of semen ejaculate, period of sexual abstinence, duration of infertility, primary or secondary infertility, and history of smoking and marijuana and alcohol use.

Semen samples were examined for sperm concentration, motility and morphology, seminal leukocyte levels via peroxidase or the Endtz test, reactive oxygen species by chemiluminescence assay, and sperm DNA damage by TUNEL assay. The patients were divided into three groups based on their seminal leukocyte levels: group 1, no seminal leukocytes; group 2, men with low-level leukocytospermia, defined as 0.1-1.0 × 10 ${ }^{6} \mathrm{WBC} / \mathrm{mL}$; and group 3, frank leukocytospermia, defined by the WHO as $>1.0 \times 10^{6} \mathrm{WBC} / \mathrm{mL}$.

\section{Semen analysis}

Computer-assisted semen analysis was performed in our andrology laboratory using a Hamilton-Thorne-Integrated Visual Optical System, version 10, semen analyzer (Hamilton Thorne Biosciences, Beverly, MA). A $5-\mu \mathrm{L}$ aliquot was loaded onto a counting chamber for each semen parameter analyzed, and 4-10 fields were examined manually and by computer-assisted semen analysis. Sperm concentration, motility and morphology using the strict criteria of Kruger et al. were reported according to $2010 \mathrm{WHO}$ criteria [1].

\section{Seminal leukocyte quantification}

To distinguish the WBCs from immature sperm, only samples with $>5$ round cells in high power field (hpf) were assessed with peroxidase staining (Endtz test) [20]. Peroxidase-positive leukocytes staining brown were counted using a Makler's counting chamber (Sefi Medical, Haifa, Israel) under bright-field microscopy and results reported as $\times 10^{6} \mathrm{WBC} / \mathrm{mL}$ semen.

\section{Reactive oxygen species}

Production of ROS was measured by the chemiluminescence assay method using luminol (5-amino-2,3-dihydro1,4-phthalazinedione; Sigma Chemical, St. Louis, MO) as the probe. Ten microliters of $5 \mathrm{mmol} / \mathrm{L}$ luminol prepared in dimethyl sulfoxide (Sigma Chemical) were added to $400 \mu \mathrm{L}$ of the washed sperm suspension. Levels of ROS were determined by measuring chemiluminescence with an Autolumat LB 953 luminometer (AutoLumat Plus LB 953, Oakridge, TN) in the integrated mode for 15 minutes. The results were expressed as Relative Light Units/sec/ $10^{6}$ sperm (hereafter referred as RLU/s) [21].

\section{Measurement of sperm DNA damage}

Sperm DNA damage was quantified using the terminal deoxynucleotidyl transferase (TdT)-mediated fluoresceindUTP nick end labeling (TUNEL) assay kit (Apo-Direct; Pharmingen, San Diego, CA) [22]. This is based on the principal that TdT binds to the 3 '-hydroxyl $(\mathrm{OH})$ termini of the single and double strand DNA breaks. It is labeled with, fluorescein isothiocyanate tagged 2'-deoxyuridine, 5 '-triphosphate nucleotides (FITC-dUTP) followed by flow cytometry. Briefly, after fixation with $3.7 \%$ paraformaldehyde for 30 minutes on ice, spermatozoa were washed and resuspended in ice cold 70\% ethanol and then in phosphate buffered saline. The specimens were centrifuged at $1600 \mathrm{rpm}$ for 7 minutes, and the pellet was resuspended for 60 minutes at $37^{\circ} \mathrm{C}$ in $50 \mu \mathrm{L}$ of a staining solution containing terminal deoxynucleotidyl transferase (TdT) enzyme, TdT reaction buffer, FITC-dUTP and distilled water. Both negative (without $\mathrm{TdT}$ ) and positive samples (DNase I) were included. After centrifugation, the cells were washed twice in rinse buffer, resuspended in $0.5 \mathrm{~mL}$ of propidium iodide (PI)/ RNase solution, and incubated for 30 minutes in the dark at room temperature in anticipation of flow cytometry [23]. PI stains total DNA and FITC-dUTP stains apoptotic cells. 


\section{Flow cytometry analysis}

All fluorescence signals of labeled spermatozoa were analyzed by the flow cytometer FACScan (Becton Dickinson). A minimum of 10,000 spermatozoa were examined for each assay at a flow rate of $<100$ cells/second. The sperm population was gated using $90^{\circ}$ and forward-angle light scatter to exclude debris and aggregates. The excitation wavelength was $488 \mathrm{~nm}$ supplied by an argon laser at $15 \mathrm{~mW}$. PI red fluorescence (580-630 nm) was analyzed in the FL-2 channel. The percentage of PIpositive cells and the mean fluorescence were calculated on a 1023-channel scale and analyzed using the flow cytometer software FlowJo, version 6.4.2 (FlowJo, LLC, Ashland, OR) [23].

\section{Statistical analysis}

Group comparisons were performed with respect to categorical variables using chi-square or Fisher's exact tests. Comparisons of greater than 2 groups with respect to quantitative variables were performed with Kruskal-Wallis tests, while 2-group comparisons were performed using the Wilcoxon Rank Sum test [24]. $P<0.05$ was considered statistically significant.

\section{Results}

Between January 2012 and April 2013, 472 infertile patients presented to the Cleveland Clinic Center for Male Fertility; inclusion and exclusion criteria are shown in Figure 1. Two hundred and eleven eligible patients were examined. Endtz testing categorized these patients as follows: group 1, no leukocytospermia $(\mathrm{n}=153)$; group 2, low-level leukocytospermia $(n=36)$, and group 3 , frank leukocytospermia, greater than $1 \times 10^{6} \mathrm{WBC} / \mathrm{mL}(\mathrm{n}=22)$. Compared to the group $1(0.0 \pm 0.01)$, the leukocyte count was significantly different in group $2(0.54 \pm 0.22 ; \mathrm{P}<$ $0.001)$ and group $3(3.78 \pm 3.08 ; \mathrm{P}<0.001)$ and between group 2 and group $3(\mathrm{P}<0.001)$. There were no significant differences between the three groups in terms of baseline clinical characteristics (Table 1) and conventional semen parameters (concentration, motility, and morphology) (Table 2).

\section{ROS levels}

Compared to $63.2 \%$ of patients with ROS levels above our reference value ( $>93 \mathrm{RLU} / \mathrm{s} / 10^{6}$ sperm), the incidence increased to $81.6 \%$ in group $2(\mathrm{P}<0.016)$ and $92.3 \%$ in group $3(\mathrm{P}<0.011)$ and it was comparable between group 2 and

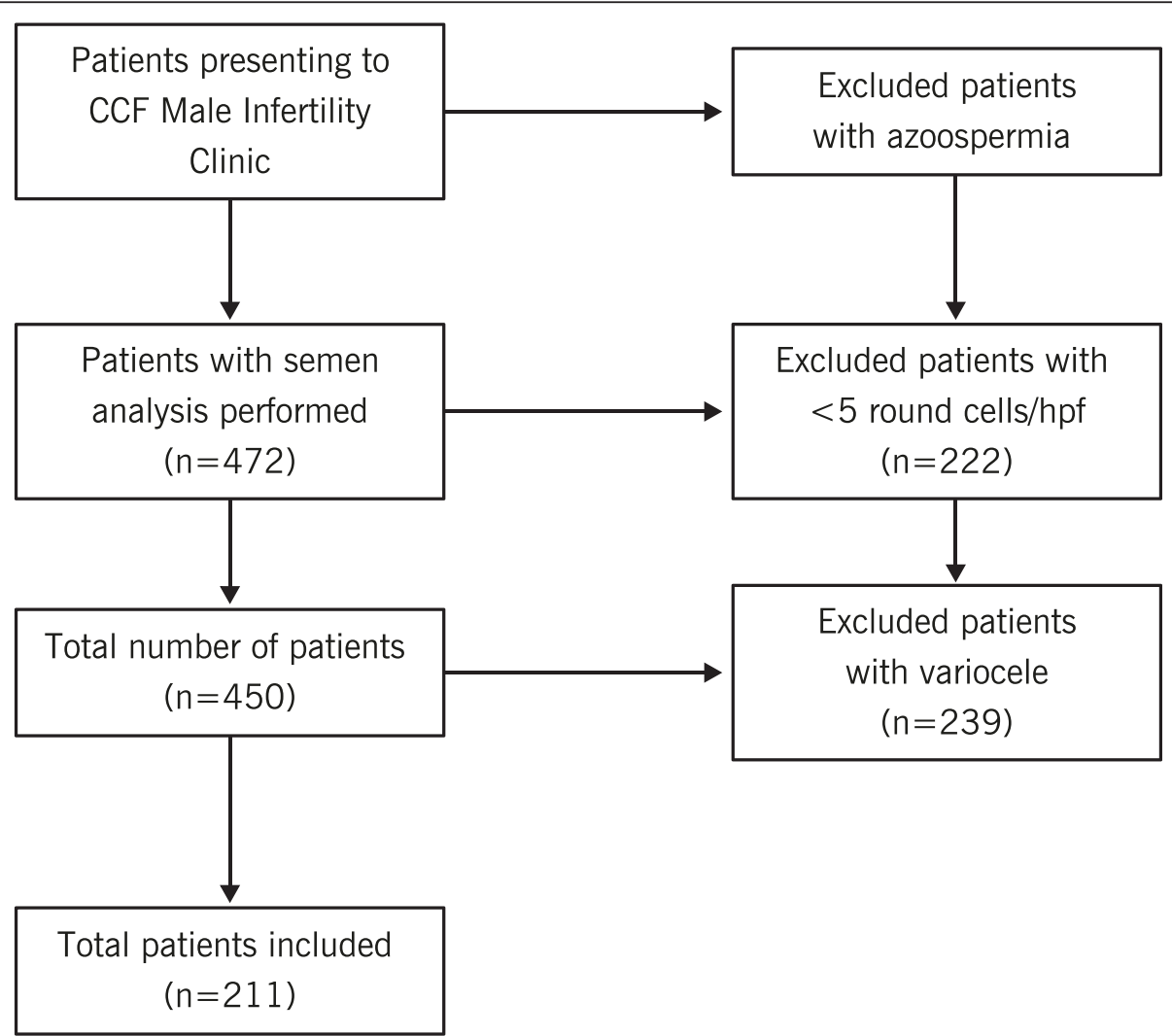

Figure 1 Flow chart demonstrating inclusion and exclusion criteria. This chart demonstrates that while 472 patients initially presented with infertility, only 211 were included in this study. 22 were excluded due to too few round cells present in their semen, and 239 patients were excluded due to their history of varicocele. Patients included were of reproductive age, and had been deemed infertile between 1-12 years. 
Table 1 Baseline clinical data in three groups of patients categorized by their seminal leukocyte levels

\begin{tabular}{|c|c|c|c|c|c|}
\hline & & $\begin{array}{c}\text { Group } 1 \\
\text { none }\end{array}$ & $\begin{array}{c}\text { Group } 2 \\
0.1-1 \times 10^{6} \mathrm{WBC} / \mathrm{mL}\end{array}$ & $\begin{array}{c}\text { Group 3 } \\
>1 \times 10^{6} \mathrm{WBC} / \mathrm{mL}\end{array}$ & $P$ \\
\hline \multicolumn{2}{|l|}{ Patients } & 153 & 36 & 22 & \\
\hline \multicolumn{2}{|l|}{ Age/years } & $36.95 \pm 6.72$ & $38.92 \pm 8.13$ & $39.05 \pm 7.41$ & 0.34 \\
\hline \multicolumn{2}{|l|}{ BMl kg/m² } & $29.49 \pm 5.17$ & $28.33 \pm 4.71$ & $28.55 \pm 4.65$ & 0.21 \\
\hline \multicolumn{2}{|c|}{ Volume of Seminal Ejaculate $(\mathrm{mL})$} & $3.08 \pm 1.32$ & $2.91 \pm 1.69$ & $2.87 \pm 1.26$ & 0.33 \\
\hline \multicolumn{2}{|l|}{ Abstinence (days) } & $4.03 \pm 1.92$ & $4.71 \pm 3.64$ & $3.41 \pm 1.18$ & 0.25 \\
\hline \multicolumn{2}{|c|}{ Infertility Duration (y) } & $2.22 \pm 1.76$ & $3.03 \pm 2.65$ & $2.50 \pm 2.39$ & 0.37 \\
\hline \multirow[t]{2}{*}{ Infertility Status } & Primary & 130 & 24 & 15 & \multirow[t]{2}{*}{0.012} \\
\hline & Secondary & 22 & 12 & 7 & \\
\hline \multirow[t]{2}{*}{ Smoking Status } & Yes & 37 & 7 & 1 & \multirow[t]{2}{*}{0.09} \\
\hline & No & 113 & 27 & 21 & \\
\hline \multirow[t]{2}{*}{ Marijuana Status } & Yes & 6 & 4 & 1 & \multirow[t]{2}{*}{0.21} \\
\hline & No & 147 & 32 & 21 & \\
\hline \multirow[t]{2}{*}{ Alcohol Status } & Yes & 93 & 18 & 14 & \multirow[t]{2}{*}{0.5} \\
\hline & No & 53 & 16 & 8 & \\
\hline
\end{tabular}

Group 1 = no leukocytospermia; Group 2 = low level leukocytospermia, Group 3 = frank leukocytospermia, all numbers are reported as mean \pm SD.

group 3. Patients with low-level leukocytospermia (group 2) had significantly higher ROS levels $(\mathrm{p}=0.001)$ and DNA damage $(\mathrm{p}<0.05)$ than patients with no leukocytospermia (group 1) (Table 2). Group 3 (frank leukocytospermia) also had significantly higher ROS levels than group 2 and group 1. Figure 2 demonstrates the differences in ROS levels between the three patient groups.

\section{DNA fragmentation}

DNA damage was measured by flow cytometry, using (FITC-dUTP). These results are shown in Table 2. At a reference value of $19 \%$ DNA damage, the incidence of DNA damage increased from $39.2 \%$ in group 1 to $50 \%$ both in group 2 and group 3. However this was not statistically significant. Higher levels of DNA damage was seen in the $0.1-1 \times 10^{6} \mathrm{WBC} / \mathrm{mL}$ group. Deoxyuridine triphosphate (dUTP) is the substrate that is added by the TdT enzyme to the free 3 ' $-\mathrm{OH}$ break-ends of DNA.
DNA damage is measured by gating the population of cells as negative or positive. This is indicated by the percentage distribution of cells that are negative or positive for DNA damage. Figure 3 is a representative example included to illustrate to illustrate the percentage DNA damage in a negative and a positive sample.

\section{Discussion}

In this study only the laboratory parameters were examined and we did not categorize the patients based on their clinical diagnosis. Reactive oxygen species are produced both by morphologically abnormal spermatozoa as well as by white blood cells - especially the granulocytes. Round cells are examined on every sample as part of the semen analysis. According to the WHO guidelines (both 1999 and 2010), round cells are tested for leukocytospermia only if the round cell number on a wet smear is higher than 5 round cells in high power field or

Table 2 Describing differences in semen parameters, ROS, and DNA damage between the 3 groups of patients

\begin{tabular}{|c|c|c|c|c|}
\hline & \multicolumn{4}{|c|}{ Leukocytospermia $\left(\times 10^{6} \mathrm{WBC} / \mathrm{mL}\right)$} \\
\hline & None & $0.1-1$ million & $>1$ million & $\mathbf{P}$ \\
\hline Leukocytes $\left(\times 10^{6} \mathrm{wbc} / \mathrm{mL}\right.$ semen $)$ & $0.0 \pm 0.01$ & $0.54 \pm 0.22^{a}$ & $3.78 \pm 3.08^{\mathrm{a}, \mathrm{b}}$ & $P<0.01$ \\
\hline Concentration $\left(\times 10^{6} / \mathrm{mL}\right)$ & $53.04 \pm 56.76$ & $69.04 \pm 80.72$ & $39.35 \pm 39.98$ & 0.97 \\
\hline Motility (\%) & $48.37 \pm 17.42$ & $47.33 \pm 25.74$ & $49.23 \pm 19.56$ & 0.8 \\
\hline Normal morphology (\%) & $3.42 \pm 3.12$ & $3.56 \pm 3.16$ & $4.14 \pm 3.79$ & 0.3 \\
\hline \multirow[t]{2}{*}{ ROS (RLU/ sec) } & 116.7 & 944.8 & 61286.8 & $<0.001$ \\
\hline & $(49 ; 550.3)$ & $(127 ; 3315.4)^{a}$ & $(6905 ; 234876)^{a, b}$ & \\
\hline DNA damage (\%) & $19.89 \pm 17.31$ & $26.47 \pm 19.64^{\mathrm{a}}$ & $24.60 \pm 17.47$ & 0.038 \\
\hline
\end{tabular}

The results are presented as mean \pm SD for all the parameters except ROS which is presented as median $\left(25^{\text {th }} ; 75^{\text {th }}\right.$ percentile).

${ }^{\mathrm{a}} \mathrm{P}<0.05$ statistically significant compared to non-leukocytospermic group.

${ }^{\mathrm{b}} \mathrm{P}<0.05$ statistically significant compared to low-level leukocytospermic group. 


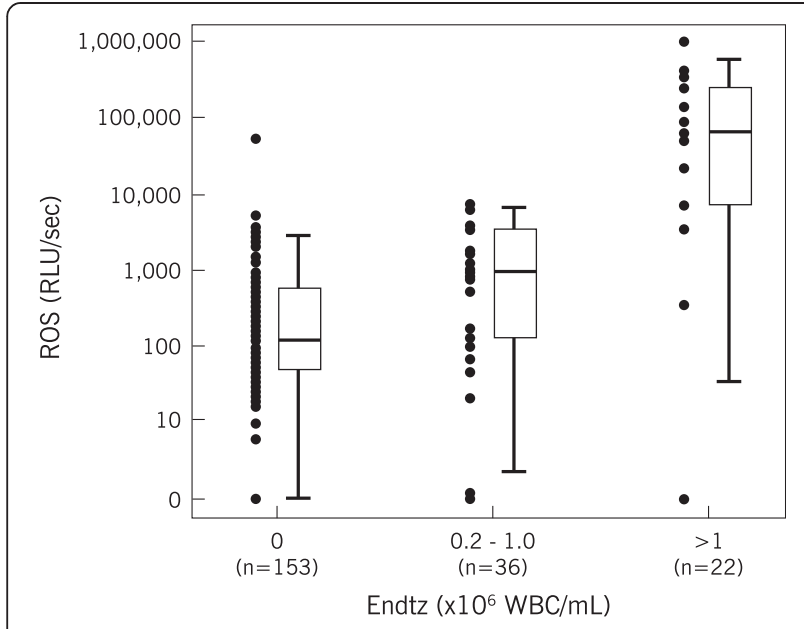

Figure 2 Box plot demonstrating minimum, maximum, median, and upper and lower quartiles of ROS data. This box-plot demonstrates the differences in ROS median, range, and upper and lower quartiles. It demonstrates that the median of the leukocytospermic group (median: 1,286.8 RLU/sec) is much higher than both the low leukocytospermic group, and the non-leukocytospermic group. Additionally, the median of the low leukocytospermic group (median: 944.8 RLU/sec) is also much higher than the nonleukocytospermic group (median: 116.7 RLU/sec).

greater than $1 \times 10^{6}$. We examined the effects of lowlevel leukocytospermia $\left(0.1-1.0 \times 10^{6} \mathrm{WBC} / \mathrm{mL}\right)$ on sperm concentration, motility and morphology as well as on oxidative stress markers of sperm (ROS levels and DNA damage). We found that while there were no significant changes in semen parameters between the 3 study groups, ROS levels and DNA damage were significantly higher in the low-level leukocytospermia group as compared to the non-leukocytospermia group. This suggests that levels of leukocytospermia lower than the current WHO threshold may impact fertility and may require treatment. It is established that the leukocytes especially the granulocytes are capable of producing significantly higher levels of ROS (>1000 fold) compared to morphologically abnormal spermatozoa with excessive residual cytoplasm in the mid piece $[25,26]$. Our study supports the earlier reports that show that low levels of leukocytospermia could also generate $\operatorname{ROS}[16,17,27,28]$ and it may have pathological effects $[14,18,19]$.

The age in the 3 groups was similar as shown in Table 1 . ROS is secreted predominantly by leukocytes, particularly the granulocytes [26]. Morphologically abnormal spermatozoa with mid-piece defects such as presence of extracellular residual cytoplasm also produce ROS. As shown in Table 2, the highest levels of ROS were seen in the leukocytospermic group. In this study we reported only Kruger's strict morphology criteria and not WHO criteria which include head, midpiece defects and tail abnormalities. Certainly prophylactic measures such as antibiotics may be prescribed to these patients even in cases of moderate leukocytospermia. The patient can be asked to provide 1) another semen sample, 2) undergo a semen culture test and reevaluating a second

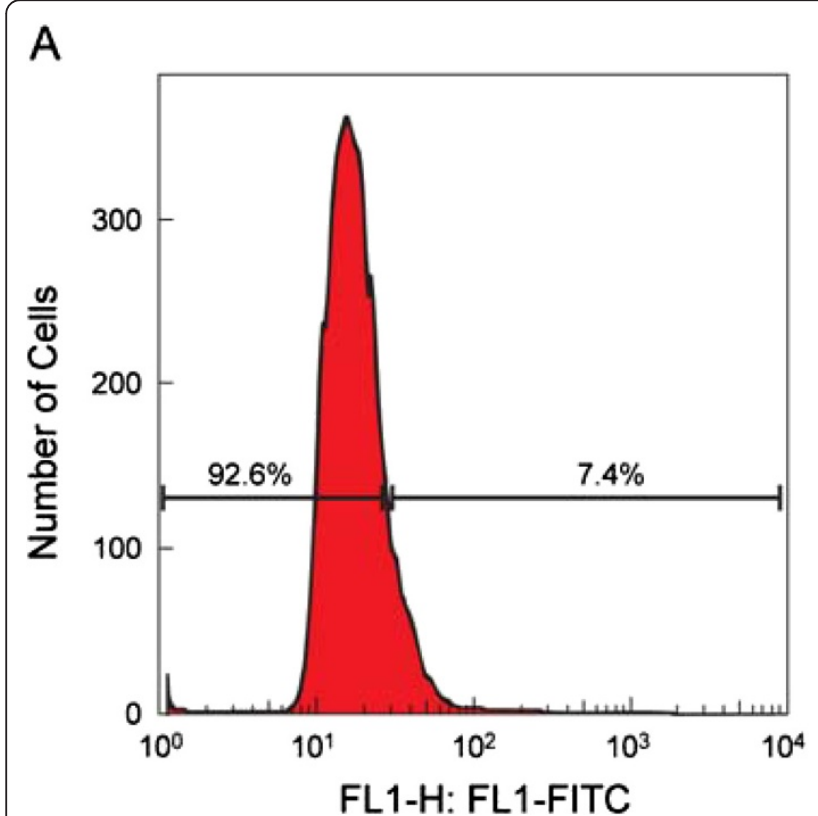

B

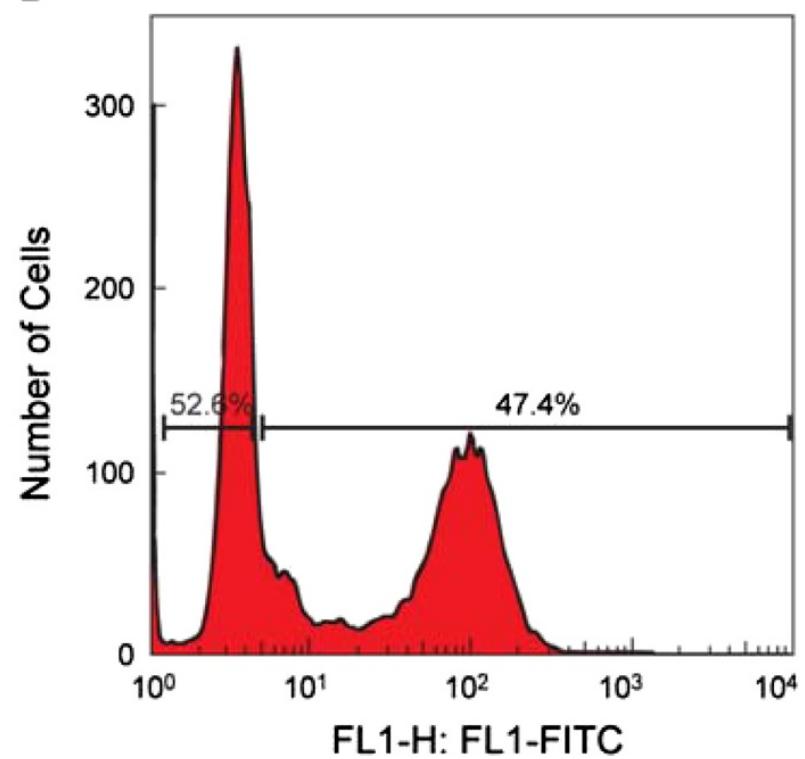

Figure 3 Flow cytometry results for DNA fragmentation. DNA damage was measured by flow cytometry, FITC-deoxyuridine triphosphate (dUTP) substrate was added to the TdT enzyme binding with the free 3'-OH termini of the single - and double strand DNA. DNA damage was measured by gating the population of cells as negative or positive. PI stains total DNA and FITC-dUTP stains apoptotic cells. A: Representative histogram for a sample that is negative for DNA damage and $\mathbf{B}$ : Representative histogram for a sample tested positive for DNA damage by flow cytometry. 
semen sample is important to see if infection has resolved and leukocyte results are negative.

The WHO establishes the threshold of $1 \times 10^{6} \mathrm{WBC} /$ $\mathrm{mL}$ as a pathological level to consider treatment of leukocytospermia. Hamada et al. showed that a 3 week course of doxycycline treatment (100 g orally, twice daily) of infertile men with leukocyte levels of $0.2-1 \times 10^{6} \mathrm{WBC} / \mathrm{mL}$ resulted in significant increases in fertilization rates of patient groups, with pregnancy rates of almost $50 \%$ in leukocytospermic samples [29]. Treating infertile men with leukocytospermia has had variable results. Treatment with antioxidants such as a combination of vitamin $E$, vitamin $\mathrm{C}$, beta-glucan, papaya, and lactoferrin resulted in a decrease in sperm DNA fragmentation [30]. Diet rich in antioxidants $\beta$-carotene $(5000 \mathrm{IU})$, vitamin $\mathrm{C}(60 \mathrm{mg})$, vitamin $\mathrm{E}(30 \mathrm{UI})$, and zinc $(15 \mathrm{mg})$ for at least 3 months as well as fruits and vegetables rich in $\beta$-carotene resulted in a significant decrease in ROS level and improvement in sperm DNA integrity in couples with previously reported high ROS and DNA damage. This also resulted in higher pregnancies and fewer miscarriage or abortion rates [31]. Lackner et al. showed that Cox-2 inhibitor therapy improves the sperm concentration and may reduce leukocyte counts in men with abacterial leukocytospermia [32]. Additionally, Yadav et al. showed that treatment with a combination of antibiotic and antioxidant treatment reduced the leukocyte count, significantly improved results of the hypo-osmotic swelling test, and significantly increased the total antioxidant capacity [33]. In that study, there was also significant reduction of nitric oxide $(\mathrm{NO})$ in the seminal plasma.

Henkel et al. examined sperm with and without DNA damage in relation to their success with IVF as well as ICSI [12]. Their results showed a significant correlation between peroxidase-positive cells and ROS, between ROS production and DNA damage, and a tendency towards lower pregnancy rates in women fertilized by DNA-damaged sperm [12]. The effects of leukocytospermia on fertility are still controversial. However, a previous study has shown that high levels of leukocytes in semen resulted in early pregnancy loss with IVF and ICSI as well as in ectopic pregnancies [13]. Both of these are pathologies that are serious to the obstetric patient population, resulting in physical and psychological damage. They were both linked more likely to a male factor, rather than a female factor [13]. While studies specifically linking leukocytospermia to pregnancy are limited, the few available show a relationship. Ignoring the possibility that low-level leukocytospermia has a pathologic effect on male fertility may neglect infertile men with this condition. In fact, low-level leukocytospermia may require testing of semen cultures for possible infection and subsequent treatment to ensure future fertility in some infertile couples.
Leukocytospermia has many implications that may not yet be entirely clear. It is shown to have a relationship with oxidative stress and sperm damage, and an eventual effect on male fertility. Lowering the accepted pathologic level of leukocytospermia may pave the way for infertile men who do not meet WHO standards to receive treatment and may give them a chance to bear biological children. As mentioned, there are already multiple avenues for treatment. These men simply need a cutoff that is more accurate and up to date. ROS are produced mainly by granulocytes [20]. In this study we demonstrated that all samples that were positive for white blood cells, as confirmed by the peroxidase or the Endtz test, were also positive for ROS. Interestingly, we did not see a significant difference in DNA damage in samples from group 2 compared to group 3 even though the levels of ROS were significantly higher in group 3 . The plausible reasons for a lack of significant difference between the two groups are: 1) in addition to oxidative stress being a major contributor of DNA damage, apoptosis also contributes to DNA damage. We did not measure apoptosis and also did not examine the clinical diagnosis in these patients. They were categorized solely on the presence or absence of leukocytes. The lack of a significant increase in DNA damage in group 3 compared to group 2 may be attributed to the patient distribution in these two groups; these patients may have adequate antioxidant reserves.

We did not measure the total antioxidant capacity (TAC) in this study. DNA damage caused by apoptosis may not translate into DNA damage as measured by Tunel assay [34]. This is because 1) unlike the somatic cell, spermatozoa nucleus is separated by the neck and the mid-piece, so apoptosis markers cannot reach the nuclear compartment [35]; 2) spermatozoa possess highly truncated base excision repair pathways which are not recognized by the 3-hydroxyl group targeted by terminal transferases in Tunel reaction and 3) nucleus is highly compacted and mitochondria activated endonucleases cannot enter the nuclear compartment. Furthermore spermatozoa do not possess apurinic/apyrimidinic (AP) endonuclease 1 (APE1) to create 3-OH breaks. Therefore Tunel signals are not generated and therefore the damage does not translate into DNA damage.

There was a 100\% correlation between Endtz positive sample and ROS production. Earlier we reported ROSTAC score which is a much stronger predictor of oxidative stress compared to ROS and TAC alone [17,27]. Leukocytospermic contamination is also associated with a reduced ROS-TAC score. It is difficult to establish a safe minimum WBC count as even low concentration can generate ROS and cause DNA damage $[16,17,28,34,36]$. We concluded that the presence of any WBCs is associated with oxidative stress and that these may therefore 
impair fertility. Complete removal of WBCs from semen samples used for assisted reproduction may help reduce oxidative stress. We had suggested that the WHO defined cutoff of $1 \times 10^{6} \mathrm{WBC} / \mathrm{mL}$ for leukocytospermia may be too high [16]. As shown in Table 2, both ROS levels and DNA damage was significant in the low level leukocytospermic group when compared to the non-leukocytospermic group.

The WHO cutoff has been challenged previously [14-18]. We are challenging it again, advocating that even men with lower levels of leukocytospermia may have risk factors for infertility, and may require treatment. Based on our present study as well as previous ones, we suggest a new lower threshold for leukocytospermia at $0.1-1.0 \times 10^{6} \mathrm{WBC} / \mathrm{mL}$.

This area of study is relatively new-whereas other studies have included low or moderate levels of leukocytospermia in their studies, this is the first study to our knowledge examining the effects of low-level leukocytospermia on oxidative stress markers. We did not examine the association of leukocyte concentration with infection. Studies examining the incidence of bacterial infection with leukocyte concentration are important in further discriminating the presence of low levels of leukoctyospermia and infection. Prescribing antibiotic regimen early on may be helpful in reducing the harmful effects of possible or mild infection. Future studies may look at specific treatment of low level leukocytospermia with antioxidants, anti-inflammatory agents, and possibly antibiotics as well as time-to-pregnancy in couples with low-level leukocytospermia.

The strengths of our study include a sizeable study population. However, our research did not show any significance between non-leukocytospermia patients and those with low level leukocytospermia in terms of DNA damage and we have explained the reason for this lack of significant increase. We expected to see a relationship here, and it was not evident. Further research may need to be undertaken to understand why differences in ROS were significant, but differences in DNA damage were not.

\section{Conclusions}

In conclusion, ROS levels and DNA damage were significantly increased in patients with low-level leukocytospermia. This tells us that there is an important implication to even a reduced number of leukocytes in the semen. We believe that low-level leukocytospermia is pathological and that treatment should be considered in these individuals in addition to semen cultures for underlying infection. The WHO guidelines for leukocytospermia may be too high and may require adjustment.

\section{Abbreviations}

BMI: Body mass index; FITC: Fluorescein isothiocynate; HPF: High power field; ROS: Reactive oxygen species; WBC: White blood cells; WHO: World Health Organization.

\section{Competing interests}

The authors declare that they have no competing interests.

\section{Authors' contributions}

AA conceived the idea, supervised the study, and edited the article for submission. SA conducted the study and helped with the data collection and management of this study. AM assisted with data analysis, writing of manuscript, and preparation for submission. MA and AMA helped with the review and editing of the manuscript. RS and ES helped with the reviewing and editing of the manuscript. All authors read and approved the final manuscript.

\section{Acknowledgement}

This work was supported by funds from the Center for Reproductive Medicine, Cleveland Clinic. The work by MA was supported by grant no. 13-MED2190-03 and APR 34-210 from KACST.

\section{Author details}

${ }^{1}$ Center for Reproductive Medicine, Cleveland Clinic, Cleveland, OH 44195, USA ${ }^{2}$ Salman Bin Abdul Aziz University, College of Medicine, Al Kharj, Jeddah, Saudi Arabia. ${ }^{3}$ Center of Excellence in Genomic Medicine Research, King AbdulAziz University, Jeddah, Saudi Arabia. ${ }^{4}$ KACST Technology Innovation Center in Personalized Medicine, King AbdulAziz University, Jeddah, Saudi Arabia.

Received: 18 September 2014 Accepted: 14 December 2014 Published: 19 December 2014

\section{References}

1. WHO laboratory manual for the examination and processing of human semen. Geneva, Switzerland: World Health Organization; 2010.

2. Wolff H: The biologic significance of white blood cells in semen. Fertil Steril 1995, 63:1143-1157

3. Esteves SC: Effect of cigarette smoking on levels of seminal oxidative stress in infertile men: a prospective study. Int Braz J Urol 2002, 28:484-485.

4. Saleh RA, Agarwal A, Sharma RK, Nelson DR, Thomas AJ Jr: Effect of cigarette smoking on levels of seminal oxidative stress in infertile men: a prospective study. Fertil Steril 2002, 78:491-499.

5. Maneesh M, Dutta S, Chakrabarti A, Vasudevan DM: Alcohol abuse-duration dependent decrease in plasma testosterone and antioxidants in males. Indian J Physiol Pharmacol 2006, 50:291-296.

6. Cocuzza M, Athayde KS, Agarwal A, Sharma R, Pagani R, Lucon AM, Srougi M, Hallak J: Age-Related Increase of Reactive Oxygen Species in Neat Semen in Healthy Fertile Men. Urology 2008, 71:490-494.

7. Griveau JF, Lannou DL: Reactive oxygen species and human spermatozoa: physiology and pathology. Int J Androl 1997, 20:61-69.

8. Jones R, Mann T: Lipid peroxides in spermatozoa; formation, role of plasmalogen, and physiological significance. Proc R Soc Lond B Biol SCi 1976, 193:317-333.

9. Villegas J, Schulz M, Soto L, Iglesias T, Miska W, Sánchez R: Influence of reactive oxygen species produced by activated leukocytes at the level of apoptosis in mature human spermatozoa. Fertil Steril 2005, 83:808-810.

10. De Lamirande $E$, Gagnon C: Reactive oxygen species and human spermatozoa. J Androl 1992, 13:379-386.

11. Pentyala S, Lee J, Annam S, Alvarez J, Veerraju A, Yadlapalli N, Khan SA: Current perspectives on pyospermia: a review. Asian J Androl 2007, 9:593-600.

12. Henkel R, Kierspel E, Hajimohammad M, Stalf T, Hoogendijk C, Mehnert C, Menkveld R, Schill W, Kruger TF: DNA fragmentation of spermatozoa and assisted reproduction technology. Reprod Biomed Online 2003, 7:477-484.

13. Barraud-Lange V, Pont J, Ziyyat A, Pocate K, Sifer C, Cedrin-Durnerin I, Fechtali B, Ducot B, Wolf JP: Seminal leukocytes are Good Samaritans for spermatozoa. Fertil Steril 2011, 96:1315-1319.

14. Punab M, Loivukene K, Kermes K, Mandar R: The limit of leucocytospermia from the microbiological viewpoint. Andrologia 2003, 35:271-278.

15. Mahfouz R, Sharma R, Thiyagarajan A, Kale V, Gupta S, Sabanegh E, Agarwal A Semen characteristics and sperm DNA fragmentation in infertile men with low and high levels of seminal reactive oxygen species. Fertil Steril 2010, 94:2141-2146 
16. Sharma RK, Pasqualotto AE, Nelson DR, Thomas AJ Jr, Agarwal A: Relationship between seminal white blood cell counts and oxidative stress in men treated at an infertility clinic. J Androl 2001, 22:575-583.

17. Saleh RA, Agarwal A, Kandirali E, Sharma RK, Thomas AJ, Nada EA, Evenson DP, Alvarez JG: Leukocytospermia is associated with increased reactive oxygen species production by human spermatozoa. Fertil Steril 2002 78:1215-1224.

18. Thomas J, Fishel SB, Hall JA, Green S, Newton TA, Thornton SJ: Increased polymorphonuclear granulocytes in seminal plasma in relation to sperm morphology. Hum Reprod 1997, 12:2418-2421.

19. Aghazarian A, Stancik I, Pfluger H, Lackner J: Influence of pathogens and moderate leukocytes on seminal interleukin (IL)-6, IL-8, and sperm parameters. Int Urol Nephrol 2013, 45:359-365.

20. Shekarriz M, Sharma RK, Thomas AJ Jr, Agarwal A: Positive myeloperoxidase staining (Endtz test) as an indicator of excessive reactive oxygen species formation in semen. J Assist Reprod Genet 1995, 12:70-74.

21. Kashou AH, Sharma R, Agarwal A: Assessment of oxidative stress in sperm and semen. Methods Mol Biol 2013, 927:351-361.

22. Sharma RK, Sabanegh E, Mahfouz R, Gupta S, Thiyagarajan A, Agarwal A: TUNEL as a test for sperm DNA damage in the evaluation of male infertility. Urology 2010, 76:1380-1386.

23. Mahfouz R, Sharma R, Lackner J, Aziz N, Agarwal A: Evaluation of chemiluminescence and flow cytometry as tools in assessing production of hydrogen peroxide and superoxide anion in human spermatozoa. Fertil Steril 2009, 92:819-827.

24. The R project for statistical computing; http://www.r-project.org

25. De Lamirande E, Leduc BE, Iwasaki A, Hassouna M, Gagnon C: Increased reactive oxygen species formation in semen of patients with spinal cord injury. Fertil Steril 1995, 63:637-642.

26. Kovalski N, de Lamirande E, Gagnon C: Reactive oxygen species generated by human neutrophils inhibit sperm motility: protective effect of seminal plasma and scavengers. Fertil Steril 1992, 58:809-816.

27. Sharma RK, Pasqualotto FF, Nelson DR, Thomas AJ Jr, Agarwal A: The reactive oxygen species-total antioxidant capacity score is a new measure of oxidative stress to predict male infertility. Hum Reprod 1999, 14:2801-2807.

28. Henkel R, Kierspel E, Stalf T, Mehnert C, Menkveld R, Tinneberg HR, Schill WB, Kruger TF: Effect of reactive oxygen species produced by spermatozoa and leukocytes on sperm functions in nonleukocytospermic patients. Fertil Steril 2005, 83:635-642.

29. Hamada A, Agarwal A, Sharma R, French DB, Ragheb A, Sabanegh ES Jr: Empirical Treatment of Low-level Leukocytospermia With Doxycycline in Male Infertility Patients. Urology 2011, 78:1320-1325.

30. Piomboni P, Gambera L, Serafini F, Campanella G, Morgante G, De Leo V: Sperm quality improvement after natural anti-oxidant treatment of asthenoteratospermic men with leukocytospermia. Asian J Androl 2008, 10:201-206.

31. Gil-Villa AM, Cardona-Maya W, Agarwal A, Sharma R, Cadavid A: Role of male factor in early recurrent embryo loss: do antioxidants have any effect? Fertil Steril 2009, 92:565-571.

32. Lackner JE, Herwig R, Schmidbauer J, Schatzl G, Kratzik C, Marberger M: Correlation of leukocytospermia with clinical infection and the positive effect of antiinflammatory treatment on semen quality. Fertil Steril 2006, 86:601-605.

33. Yadav SB, Suryakar AN, Huddedar AD, Shukla PS: Effect of antioxidants and antibiotics on levels of seminal oxidative stress in leukocytospermic infertile men. Indian J Clin Biochem 2006, 21:152-156.

34. Aitken RJ, Baker MA: Causes and consequences of apoptosis in spermatozoa; contributions to infertility and impacts on development. Int J Dev Biol 2013, 57:265-272.

35. Koppers AJ, Mitchell LA, Wang P, Lin M, Aitken RJ: Phosphoinositide 3-kinase signalling pathway involvement in a truncated apoptotic cascade associated with motility loss and oxidative DNA damage in human spermatozoa. Biochem J 2011, 436:687-698.

36. Aitken RJ, Jones KT, Robertson SA: Reactive oxygen species and sperm function-in sickness and in health. J Androl 2012, 33:1096-1106.

doi:10.1186/1477-7827-12-126

Cite this article as: Agarwal et al:: Reactive oxygen species and sperm DNA damage in infertile men presenting with low level

leukocytospermia. Reproductive Biology and Endocrinology 2014 12:126.

\section{Submit your next manuscript to BioMed Central and take full advantage of:}

- Convenient online submission

- Thorough peer review

- No space constraints or color figure charges

- Immediate publication on acceptance

- Inclusion in PubMed, CAS, Scopus and Google Scholar

- Research which is freely available for redistribution 